\title{
Las habilidades sociales en la adolescencia temprana: perspectivas desde la Psicología Positiva
}

E. Norma Contini de González*

\section{Resumen}

El objetivo de este trabajo es hacer referencia a las habilidades sociales (HHSS) en la adolescencia temprana.

El interés en el tema obedece a que el éxito personal y social parece estar más vinculado con las habilidades interpersonales, que con las habilidades cognitivas expresadas en medidas sintéticas de coeficiente intelectual (CI); porque las HHSS constituyen una de las mayores fuentes de autoestima y bienestar personal.

Se deslindan los conceptos de inteligencia social, habilidades sociales, competencia social, asertividad y comportamiento adaptativo. Se caracterizan las HHSS y se describen los estilos de habilidades sociales encontrados. Se hace referencia a teorías explicativas de las mismas. Se analizan las posibles interacciones entre HHSS, personalidad y ajuste psicosocial. Se conceptualiza la Psicología Positiva y se argumenta porqué las HHSS constituyen parte del capital psíquico del niño/adolescente. Finalmente, se hacen consideraciones sobre la importancia de estudiarlas en la adolescencia temprana. El diagnóstico oportuno de los recursos con los que cuenta el adolescente -o bien sus disfunciones, como la agresividad o el aislamiento- permitirá delinear programas de intervención que promuevan habilidades protectoras para su desarrollo y que favorezcan su permanencia en el sistema escolar, como forma de inclusión social. Se concluye argumentado que las HHSS operan como un recurso salugénico en la adolescencia temprana en el marco de la Psicología Positiva.

Palabras clave: habilidades sociales - adolescencia - Psicología Positiva

\section{Abstract}

Cognitive abilities in early adolescence: an outlook from positive psychology

The aim of this work is to refer to social abilities in early adolescence.

Personal success and social success seem to be more related to interpersonal abilities than with those cognitive abilities expressed in synthetic measures of IQ. Social abilities are also one of the major sources of self esteem and personal well-being.

\footnotetext{
* Doctora en Psicología. Facultad de Psicología, Universidad Nacional de Tucumán. Investigadora CIUNT. San Juan 150, 3er. Piso, Dpto.5. 4000 S.M.de Tucumán. Argentina. Email: nocon@arnet.com.ar
} 
The concepts of social intelligence, social abilities, social competence, assertiveness and adaptative behaviour are differentiated. Social abilities are characterized. The different types of social abilities founded are described and the theories which explain those types of abilities are referred. Possible interaction between social abilities, personality and psychosocial adjustment are analyzed. Positive Psychology is defined. It is explained why the Social Abilities are part of child and adolescent's psychological capital. It is also treated why is important to study those aspects recently mentioned in early adolescence.

Opportune diagnosis of those types of adolescent resources -or their dysfunctions like aggressiveness or isolation- would allow tracing a lay out intervention programs which promote protective abilities for its development which also would help the permanence in the scholar system as a way of social inclusion. Finally, it is explained how social abilities work as a salugenic resource in early adolescence within the frame of Positive Psychology.

Key words: social abilities- adolescence- Positive Psychology. 
El siglo XX ha sido testigo de innumerables investigaciones sobre la inteligencia, lo que trajo como consecuencia una mejor definición del constructo y un considerable refinamiento metodológico para su evaluación. A su vez, la práctica clínica y psicopedagógica fue mostrando la complejidad del funcionamiento cognitivo de un sujeto y las dificultades para establecer pronósticos certeros sobre el éxito en la vida cotidiana a partir de las pruebas de CI. Así, sujetos con elevado CI tienen dificultades en su desempeño en la vida diaria, mientras otros, de CI modesto, lo hacen sorprendentemente bien.

¿Qué factores entran en juego para producir el éxito o fracaso en la vida cotidiana?

Sobre la base de este interrogante, en las últimas dos décadas el interés se ha ido centrando en las formas no académicas de la inteligencia. Así, se han utilizado distintos constructos para poder definirlas. Uno es el de Inteligencia Práctica (Sternberg 1985, 1997); otro concepto alternativo es el de inteligencia social (Cantor y Kihlstrom, 1987; Ford \& Maher, 1998), habilidades de interacción social (Monjas Casares, 2000) o el de las inteligencia intrapersonal e interpersonal (Gardner, 1994, 1993), el de inteligencia emocional (IE) (Goleman, 2000; Salovey y Mayer, 1990) y el de Conocimiento Tácito (Neisser, 1979; Polanyi, 1966; Schön, 1983; Sternberg, 1985, 1997).

Se hará referencia a las habilidades de interacción social y se analizará la importancia que han cobrado los estudios sobre las mismas, particularmente en la infancia y adolescencia temprana. Tal relevancia se vincula al impacto que parece tener en la organización psicológica del adolescente, en la calidad de vida, en la inclusión escolar y social del mismo. Desde esta perspectiva se argumentará que las HHSS pueden considerarse dentro del paradigma de la Psicología Positiva.

\section{Inteligencia social versus inteligencia académica}

Las investigaciones sobre la inteligencia académica se han realizado profusamente a lo largo del siglo XX, mientras que las referidas a la inteligencia emocional (IE) y social son recientes. Tradicionalmente, se pensó que las oportunidades de éxito de un sujeto dependían de la inteligencia académica sintetizada en el CI.

Aun hoy se afirma, que existe una relación entre la capacidad del sujeto - evaluada por pruebas de CI - y su desempeño, es decir, sujetos con CI bajo se posicionan en trabajos de baja jerarquía, mientras que los de CI alto suelen ocupar cargos bien remunerados. Pero esto no es una ley infalible y se puede aseverar que las pruebas de CI son relativamente inadecuadas para predecir de un modo inequívoco el éxito en la vida. Goleman señala que el CI contribuye aproximadamente con un $20 \%$ a los factores que determinan el éxito en la vida. El 80\% restante estaría compuesto por otras variables inherentes a la IE, tales como: el autodominio, la capacidad para motivarse a sí mismo, perseverancia frente a las frustraciones, control de los impulsos, regulación del humor, empatía y tener esperanza.

Con respecto a las pruebas de CI se ha podido confirmar que correlacionan positivamente con el rendimiento escolar, en cambio no aseguran que las capacidades 
evaluadas por este tipo de pruebas habiliten al sujeto a resolver los problemas de la vida cotidiana. A pesar de ello, la escuela y la cultura han puesto énfasis en las habilidades académicas en desmedro de la IE, aunque esta tenga relevancia para el éxito en la vida diaria. Las investigaciones realizadas permiten afirmar que un sujeto con habilidades emocionales y sociales, que conoce y controla sus propios sentimientos, que logre interpretar los estados de ánimo de otros, podrá operar en su entorno de tal manera, que redunde positivamente en su calidad de vida.

\section{La inteligencia práctica ¿sirve para resolver problemas académicos?}

Los problemas que habitualmente los sujetos deben afrontar han sido clasificados en académicos y prácticos (Neisser, 1979; Sternberg, 1985, 1997; Wagner \& Sternberg, 1985).

Se puede afirmar que los sujetos frecuentemente tienen que encarar situaciones cuya solución no emerge del conocimiento adquirido en el contexto académico. Estos tipos de problemas se experimentan en la vida diaria. La inteligencia práctica o de la vida cotidiana se presenta así, diferente de la inteligencia académica.

La inteligencia práctica se relaciona con el sentido común y la perspicacia para captar situaciones de la calle, mientras que la inteligencia académica, supone operaciones formales del pensamiento sobre temas generalmente ajenos a la experiencia cotidiana del sujeto. Con frecuencia, se puede observar que sujetos exitosos en la escuela presentan déficit en sus relaciones sociales, o a la inversa, personas que fracasan en la escuela tienen éxito en el trabajo o en los vínculos sociales.

Sternberg señala, con mucho acierto, que las habilidades en las que enfatiza la escolarización formal tienen un valor muy limitado si no pueden ser empleadas en la resolución de problemas de la vida diaria. En tal sentido, las críticas a la escuela han sido muy severas, puesto que parece no poder resolver la disociación entre el saber académico del aula, y el de la vida cotidiana.

\section{Las habilidades de interacción social}

El estudio de las habilidades de interacción social (HHSS) ha cobrado auge en las últimas décadas (Ballester \& Gil LLario, 2002; Gil \& León Rubio, 1998; Monjas Casares, 2000). Se han generado numerosas investigaciones y sobre la base de dichos resultados, se han diseñado variados programas de intervención para aumentar la competencia social o las habilidades sociales, que son algunas de las denominaciones con las que se las identifica.

El interés por el tema se vincula al hecho indiscutible de que los sujetos pasan gran parte de su tiempo con otros, en interacción. Esta necesidad de vínculos interpersonales se asocia al hecho de que las relaciones sociales positivas son una de las mayores fuentes de satisfacción y bienestar personal. Tal como señalan Ballester \& Gil Llario 
(2002), la competencia social proporciona sentimientos de autoeficacia que constituye un ingrediente fundamental de la autoestima. Ser reconocido y aceptado por otro produce un impacto favorable en la autoestima, y ello refuerza la satisfacción vital.

Existen numerosas investigaciones que constatan la existencia de fuertes relaciones entre el desarrollo de habilidades sociales en la infancia y adolescencia y el ajuste social, psicológico y académico en la vida adulta (Monjas Casares, 2000; Hops \& Greenwood, 1988; Laad \& Asher, 1985). El desarrollo de habilidades sociales positivas en la infancia y adolescencia contribuye al logro de una personalidad saludable en la adultez. Y como contrapartida, la competencia interpersonal pobre, se asocia con baja aceptación, rechazo o aislamiento de los pares, con problemáticas escolares y con problemáticas personales, tales como la baja autoestima, la indefensión, inadaptación en la adolescencia, delincuencia juvenil o adicciones. Con respecto a las problemática escolares, la incompetencia social se vincula con bajo rendimiento, fracaso, ausentismo y expulsión de la escuela (Monjas Casares, 2000). En el particular caso de los adolescentes de contextos de pobreza este déficit en habilidades sociales va a tener un efecto devastador, pues acelera su expulsión del sistema educativo y, para estos jóvenes, la escuela sigue siendo un lugar privilegiado de inclusión social, de allí su importancia.

\section{Habilidades sociales y relación con los pares}

Nadie duda hoy de la importancia del vínculo madre-hijo en la organización psíquica del niño y, en ese marco, se incluye el desarrollo de las HHSS. La teoría psicoanalítica es la que puso el mayor énfasis en el vínculo madre-hijo, mientras que en las dos últimas décadas, sobre la base de diversas investigaciones se ha podido comprobar la importancia decisiva que tienen las relaciones entre pares en la infancia y adolescencia. El vínculo entre iguales promueve el desarrollo de habilidades de interacción social y ello impacta positivamente en la salud psíquica del sujeto.

Monjas Casares (2000) analiza pormenorizadamente las funciones que cumplen la relación con los pares en el logro de HHSS positivas. Las mismas, permiten el conocimiento de sí mismo y de los demás; el desarrollo de conductas que denomina de reciprocidad, esto es, la posibilidad de apreciar lo que se da y lo que se recibe. Alude también al intercambio en el control de la relación, con lo cual quiere significar que el niño/adolescente aprende que en ocasiones él lidera una situación y, en otras, le corresponde hacerlo a un par.

Otras funciones que destaca como esenciales son las de colaboración, es decir la posibilidad que tiene un niño/adolescente de trabajar con otros niños, facilitando la tarea en común; las estrategias sociales de negociación y de acuerdos; autocontrol de la propia conducta en función del feed-back que recibe de los otros. Agrega que la relación con los iguales, opera como apoyo emocional y como fuente de disfrute. Por último, destaca que hace posible el aprendizaje del rol sexual y el aprendizaje de valores.

De este análisis, se puede concluir que la relación con los pares es decididamente importante en el proceso de desarrollo del niño y actuaría como un factor protector 
para el logro de la salud en la adolescencia y adultez. Desde esta perspectiva se piensa que las HHSS se inscriben en el paradigma de la Psicología Positiva, tema que se abordará en apartados posteriores.

\section{¿Qué son las habilidades de interacción social?}

Tal como plantea Fernández Ballesteros (1994) existe una acentuada dificultad para acordar una definición de HHSS y esa dificultad se vincula a que están referidas siempre a un contexto específico.

En primer lugar, corresponde señalar que existe controversia en torno a la superposición de conceptos como competencia social, habilidades de interacción social, asertividad o comportamiento adaptativo.

Competencia social hace referencia a un juicio valorativo que emite un sujeto respecto al grado de adecuación del comportamiento social de otro en un contexto determinado (McFall, 1982, Monjas Casares, 2000). Quien emite ese juicio suele ser alguien significativo, por ejemplo la madre o un profesor con respecto al comportamiento de un niño o adolescente.

Por habilidades sociales se entiende en cambio, conductas especificas necesarias para ejecutar competentemente una tarea interpersonal (Monjas Casares, 2000). Se define a dichas habilidades de la infancia como: "las conductas necesarias para interactuar y relacionarse con los iguales y con los adultos de forma efectiva y mutuamente satisfactoria” (2000, p. 29). Se destaca que se hace referencia a comportamientos adquiridos, o sea, aprendidos y no a rasgos de personalidad.

Según Caballo (1993), las HHSS son un conjunto de conductas que permiten al individuo desarrollarse en un contexto individual o interpersonal expresando sentimientos, actitudes, deseos, opiniones o derechos de un modo adecuado a la situación. Generalmente, posibilitan la resolución de los problemas de la vida cotidiana. Las HHSS permiten así afrontar situaciones interpersonales, lo que las torna necesarias para la adaptación del sujeto al ambiente más próximo.

Desde una perspectiva interaccionista, la habilidad social es "la capacidad que el individuo posee de percibir, entender, descifrar y responder a los estímulos sociales en general, especialmente a aquellos que provienen del comportamiento de los demás” (Blanco, 1982, p. 568).

Ballester \& Gil Llario (2002) cuando se interrogan qué son las habilidades sociales aluden en realidad a lo que llaman un "comportamiento socialmente competente" (p. 10). Señalan que si bien es difícil definirlo, existen ciertos parámetros en los que la mayor parte de los estudiosos acuerdan, y que permiten definir dicho comportamiento. Ellos son: el consenso social, la efectividad y el carácter situacional.

Analizan estos componentes y afirman que el consenso social alude a que un comportamiento es considerado correcto, si cuenta con la aprobación del grupo. El sujeto tiende a repetir comportamientos que han sido valorados por otros sujetos. El consenso social determina que un comportamiento pueda ser considerado adecuado 
por un grupo y no por otro. Y allí radica la complejidad del tema, tal cual lo ha señalado Fernández Ballesteros. En cuanto a la efectividad, Ballester \& Gil Llario señalan que "una conducta es habilidosa en la medida que conduce a la obtención de aquello que se propone” (p. 11). Adjudican especial importancia al control emocional del sujeto, puesto que ello le permitirá expresar sus sentimientos sin ansiedad. La presencia de ansiedad, que puede identificarse, por ejemplo por el tono de voz o la actitud del sujeto deteriora el comportamiento. Y por último refieren al carácter situacional de la competencia social. Aquí, son importantes el grado de familiaridad con el interlocutor, el género y el propósito de la interacción. En función de este planteo, concluyen que no tienen fundamentos pensar que un sujeto va ser igualmente competente al emplear una misma habilidad en diferentes situaciones

León Rubio \& Medina Anzano (1998) han individualizado cuatro características que delimitan el concepto de habilidad social. Ellas son: 1) el carácter aprendido; 2) conciencia de interdependencia de la habilidad social, es decir, todo comportamiento social exige entender el punto de vista del otro; 3) los objetivos que persigue son solo comprensibles en situaciones sociales específicas; 4) la habilidad social es considerada sinónimo de eficacia en el comportamiento interpersonal. A partir de la identificación de las mencionadas características, definen a la habilidad social como "la capacidad de ejecutar aquellas conductas aprendidas que cubren nuestras necesidades de comunicación interpersonal y/o responden a las exigencias y demandas de las situaciones sociales de forma efectiva” (León Rubio \& Medina Anzano, 1998).

El término habilidades de interacción social, suele usarse como sinónimo de habilidades sociales, habilidades de relación interpersonal o competencia social. En este trabajo, se hará referencia específicamente al comportamiento interpersonal, ya que el constructo competencia social incluye otros comportamientos adaptativos, tales como vestirse, alimentarse, tomar el ómnibus y no solo la conducta interactiva.

Otro término muy empleado es el de asertividad, que ha sido utilizado para referir a conductas de autoafirmación y expresión de sentimientos. Se considera necesario analizarlo a fin de determinar el grado de superposición que puede tener con el concepto de habilidades sociales.

\section{Acerca de la asertividad}

Se entiende por asertividad: la posibilidad que tiene un sujeto de expresar adecuadamente las emociones en las relaciones sociales, es decir, que al manifestarse deben estar ausentes signos de agresividad o ansiedad (Da Dalt de Mangione \& Difabio de Anglat, 2002).

Es Wolpe (1977) quien inaugura el término, y posteriormente, se fueron haciendo reelaboraciones del concepto y consideraciones sobre los alcances del mismo. Hace referencia a la capacidad de autoafirmación en el contexto de las relaciones interpersonales. Caballo (2000) puntualiza, que la asertividad es la capacidad que tiene un sujeto para expresar pensamientos o sentimientos a otros de un modo directo, efectivo 
y apropiado. El sujeto asertivo tiene una conducta de afirmación de los conceptos u opiniones que sostiene, estando ausentes connotaciones de oposición o agresividad hacia el otro.

A su vez, el constructo asertividad se inserta en otro denominado prosocialidad (Pithod, 1993). Señala que los sujetos con motivación altruista, denominada también prosocial genuina son proclives a desarrollar una personalidad sana y asertiva.

Finalmente, cabe señalar que existe coincidencia en diversos autores (Paula Pérez, 2000; Caballo, 1993; Da Dalt de Mangione \& Difabio de Anglat, 2002; Monjas Casares, 2000) acerca de que el concepto de asertividad es restringido y que se integra dentro de otro más amplio, el de habilidades sociales.

\section{Estilos de habilidades sociales}

Si se toma como referente el concepto de asertividad, pueden identificarse tres estilos de conducta: no asertivo, agresivo y asertivo.

El estilo asertivo permite el establecimiento de relaciones sociales significativas y una mejor adaptación social (Caballo, 1993). Un sujeto asertivo sería aquel que se expresa afirmativamente, con seguridad y defiende sus derechos sin utilizar conductas agresivas. Afronta las situaciones adecuadamente, explica sus ideas dando su propia perspectiva sin desconocer las ajenas (Da Dalt de Mangione \& Difabio de Anglat, 2002). Pithod (1993) agrega que el sujeto asertivo tiene una orientación activa en la vida. Por otra parte, la resolución de conflictos está sostenida por un razonamiento lógico que predomina sobre los sentimientos.

Como contrapartida, el sujeto con estilo no asertivo se caracteriza por carecer de habilidad para defender sus derechos y para expresar sus ideas y sentimientos. Se somete a los deseos de los demás, y adopta una posición pasiva frente a los conflictos. Predomina una actitud de huida, como modo de no afrontar los mismos, y se caracteriza por la inseguridad sobre qué hacer o decir. Necesita ser apreciado por los demás, ya que considera que no es tenido en cuenta, y de allí su temor a ofender y perder la estima de los otros (Da Dalt de Mangione \& Di Fabio de Anglat, 2002). El comportamiento no asertivo produce como efecto la falta de valoración de los demás, lo cual influye negativamente en la autoestima del sujeto.

A su vez, el sujeto con estilo agresivo expresa emociones y pensamientos de manera inapropiada, sin considerar los derechos de los demás. Carece de habilidad para afrontar situaciones conflictivas, ya que lo hace con violencia verbal o física. Adopta posiciones radicales en el análisis de los hechos, lo que le impide percibir los matices de los mismos para valorarlos y para adoptar posturas equilibradas. Todo ello, lo conduce a ofuscarse cada vez más con un mayor número de personas. El comportamiento agresivo del sujeto genera rechazo por parte de los demás.

Monjas Casares (2000) señala, que el déficit en HHSS, ya sea en el polo no asertivo, como en el agresivo se presenta vinculado con escasa aceptación, rechazo o aislamiento social por parte de los pares; con bajo rendimiento escolar, expulsión de la escuela o 
inadaptación escolar; baja autoestima; desajustes psicológicos y psicopatología en la infancia, tales como la depresión; en la adolescencia se asocia con la iniciación en la delincuencia, alcoholismo, suicido o toxicomanías.

Tanto los estilos de vinculación no asertivo como agresivo dan cuenta de un déficit en las HHSS (Caballo, 1993; Connor et al., 1982; Godoy et al., 1993; Matson et al, 1983). Al respecto, se han llevado a cabo numerosos estudios a nivel internacional y algunos en Argentina sobre los déficit en las HHSS, sobre todo aquellos relacionados con el comportamiento agresivo y el comportamiento inhibido en la infancia y la adolescencia (Ayala Velásquez, 2002; Brailo, Seguel \& Montenegro, 1987; Cervone et al., 2000; Ison \& Soria, 1997; Ison, 2004; Ortega \& Monks, 2005; Samaniego, 1998, 2004). La presencia de estas disfunciones expresa un malestar que da cuenta de un contexto socio-económico y cultural que no ofrece oportunidades de crecer en salud al niño/adolescente.

\section{Características de las habilidades sociales}

Se dirá que en primer lugar, las HHSS son comportamientos aprendidos (Caballo, 1987, 1991; Kelly, 1987; Monjas Casares, 2000), siendo decisivo el entorno en el que está inserto el niño o el adolescente. La familia primero, la escuela luego, y la comunidad en un sentido amplio brinda un marco en el cual se da el proceso de aprendizaje de dichas habilidades. Según que ese contexto sea salugénico o disfuncional, el estilo de dichas habilidades será luego asertivo, inhibido o agresivo.

Monjas Casares (2000) señala, que se aprende por experiencia directa o por observación, tanto de otros sujetos como de modelos simbólicos, por ejemplo de la TV. Agrega que también se aprende, mediante el uso del lenguaje hablado, es decir, por medio de preguntas, instrucciones o sugerencias y constituiría una forma no directa de aprendizaje; por ejemplo en el contexto de la familia, los padres inducen al niño a saludar a un familiar que llega de visita, o a decir gracias cuando recibe un obsequio. Finalmente, alude al aprendizaje por feed-back interpersonal, es decir que el niño/ adolescente recibe una retroalimentación de su interlocutor que informa la cualidad de su comportamiento social; por ejemplo, la madre demostrará con una sonrisa su aprobación al ver que el niño besa a la abuela que llega de visita, o desagrado si aquél arroja al suelo un juguete que le regalan.

Otra característica es que las HHSS implican componentes verbales, motrices, emocionales y cognitivos. Igualmente las HHSS son respuestas a situaciones específicas, esto quiere decir, que dependen del contexto en el cual se producen, de las personas que intervienen, y de la situación en la cual tienen lugar. Otra particularidad, es que las HHSS implican siempre la relación con otros, ya sean pares o adultos. Lo que define la cualidad de la interacción, son las personas que participan y el contexto en el cual se da dicha interacción. Por ejemplo, no es lo mismo entablar un diálogo entre dos compañeros en el aula, que en el gimnasio o en un salón de baile; también será distinta la interacción entre dos compañeros de clase, que entre un alumno y el profesor. 


\section{Habilidades sociales, personalidad y ajuste psicosocial}

Si se revisan las definiciones de la conducta no asertiva, agresiva y asertiva detalladas precedentemente, es posible encontrar rápidamente puntos de coincidencia con algunas formulaciones que Millon (1969, 1977, 1990, 1994, 1998) denominó "patrones de personalidad”. En el caso de la población adolescente, tema que interesa en esta presentación, Millon diseña un inventario (MACI) para evaluar trastornos de personalidad en población clínica, e incluye en el mismo, diversas escalas y su correspondencia con las categorías del DSM-IV. De entre ellas se citan algunas, tales como: Escalas Inhibido (DSM-IV: Evitativo); Afligido (DSM-IV Depresivo) y Sumiso (DSM-IV: Dependiente), en cuyas descripciones se encuentran semejanzas con el estilo no asertivo de habilidades sociales. Por otra parte, en la descripción de las Escalas Transgresor (DSM-IV: Antisocial) y Poderoso (sádico) se identifican similitudes con el estilo agresivo de habilidades sociales. A partir de estos puntos de coincidencia, el interrogante circula en torno a si existe relación entre las HHSS, los patrones de personalidad y el ajuste psicológico del adolescente.

En la literatura psiquiátrica y psicológica hay referencias a que el déficit en HHSS puede generar desajustes psicológicos (Sullivan, 1953; Argyle, 1983). A su vez, existe una discusión acerca de si el déficit en HHSS es causa o consecuencia de cuadros psicopatológicos. Siguiendo a León Rubio y Medina Anzano (2002) se puede afirmar que la carencia de HHSS promueve en el sujeto, el empleo de estrategias inadecuadas para la resolución de temas y problemas de la vida cotidiana, generalmente de carácter social. Desde esta perspectiva, las HHSS adecuadas son un prerrequisito para un buen ajuste psicológico, esto es, para la salud psíquica del adolescente - que es la edad que nos interesa - mientras que no sería correcto considerar a dichas habilidades, como secundarias a un trastorno psicopatológico (Gilbert \& Connoly, 1995; Kelly, 1987).

Este cambio epistemológico, explicaría el creciente interés por la investigación y la formulación de programas de intervención en HHSS en niños y adolescentes en las últimas décadas. Los resultados de las mismas, dan cuenta del estrecho vínculo entre las HHSS en la infancia y el posterior ajuste psicosocial (Hops \& Greenwood, 1988; Laad \& Asher, 1985).

\section{Algunas teorías explicativas de las habilidades sociales}

De entre los diversos modelos explicativos de las habilidades sociales, dos resultan de interés: el modelo interactivo y el del aprendizaje social.

Según el modelo interactivo, las HHSS serían los resultados de procesos cognitivos y de conducta, en una secuencia que se inicia con la percepción correcta de estímulos interpersonales relevantes, continúa con el procesamiento flexible de estos estímulos para evaluar la mejor opción de respuesta, y concluye con la manifestación de la opción elegida (León Rubio \& Medina Anzano, 1998). El sujeto tiene en este modelo un rol activo, en cuanto busca, procesa información y 
controla sus acciones. La característica esencial (Morales, 1981) es la influencia mutua entre las dos personas que interaccionan. Trower (1986) y McFall (1982) son dos figuras significativas dentro de este modelo.

En la teoría del aprendizaje social, el esfuerzo ha estado centrado en poder explicar el comportamiento a partir del estudio experimental del aprendizaje. Figuras relevantes son Dollard \& Miller (1977). Estos investigadores confirieron importancia a la imitación como factor socializador. Pero, es Bandura, quien hará la mayor contribución al respecto al estudiar la agresión en niños (Bandura y Walters, 1978). Bandura afirma que el funcionamiento psicológico se explica por una continua interacción entre factores personales y ambientales. Esta reciprocidad, lo llevó a estudiar lo que denominó aprendizaje observacional, que consideraba no idéntico a la imitación. Plantea, que el sujeto no se limita a reproducir mecánicamente el comportamiento del otro, sino que extrae reglas generales de cómo actuar y lo pone en práctica cuando considera que con ello va obtener buenos resultados.

En síntesis, se sostiene que la conducta está determinada en gran medida por factores externos, pero también, por variables del sujeto. Éste, es capaz de controlar su comportamiento mediante metas autoimpuestas, que denomina autorreforzamiento.

\section{Las habilidades sociales en el marco de la Psicología Positiva}

En párrafos precedentes, se hizo mención a la función esencial que cumple la relación con los pares en el logro de HHSS en la infancia y adolescencia. Se señaló así, siguiendo a Monjas Casares (2000), que permiten el conocimiento de sí mismo y de los demás; el desarrollo de conductas que denomina de reciprocidad, de colaboración, estrategias sociales de negociación y de acuerdos; autocontrol de la propia conducta. Igualmente se señaló, que dichas relaciones cumplen la función de apoyo emocional, de ser una fuente de disfrute, haciendo posible también, el aprendizaje del rol sexual y el aprendizaje de valores.

Si se analizan cuidadosamente estas funciones se destaca: que ponen énfasis en aspectos saludables o salugénicos del niño/ adolescente y, desde este punto de vista entroncan con los postulados de la Psicología Positiva.

La Psicología Positiva es un campo emergente en los últimos diez años, que busca contrarrestar el excesivo énfasis que la psicología del siglo XX puso en el malestar y en la psicopatología (Vera Poseck, 2006; Vásquez, 2006; Casullo, 2007). Se considera a este enfoque - psicopatológico - como reduccionista.

El término Psicología Positiva ha sido propuesto por Seligman (2003), investigador de la Universidad de Pennsylvania, quien luego de dedicarse al estudio de las patologías mentales hace una formulación que quita el foco de aquellas y se centra en una concepción más positiva del sujeto.

Los antecedentes de la Psicología Positiva pueden encontrarse en la Psicología Humanística, con los aportes de Maslow y de Rogers. También van en el mismo sentido los hallazgos de Antonovsky (1979; 1988), quien, investigando desde el campo de la 
Sociología de la Salud en la Universidad Ben Gurion, Israel, enuncia lo que denomina el modelo salugénico como contrapuesto al clásico paradigma psicopatológico. En Unraveling the mystery of health. How people manage stress and stay well (1988) relata que, mientras analizaba datos de un estudio sobre la adaptación al climaterio de mujeres de diferentes grupos étnicos en Israel, advirtió que un grupo de ellas había estado en campos de concentración. Haciendo un análisis de la salud psíquica de aquél con relación al grupo control (mujeres que no habían estado en campos de concentración) concluyó que un 29\% de las sobrevivientes tenían un nivel de salud psíquica bastante aceptable y un razonable estado de bienestar. La pregunta giraba en torno a qué recursos tenían para que, no obstante haber pasado por la experiencia límite de la guerra, de la vida en un campo de concentración y de los años posteriores de displacer - luego de concluida la contienda- conservaran aún, un aceptable estado de bienestar. Antonovsky se propuso develar este enigma y formula lo que denominó el modelo salugénico y, dentro de éste, lo que llamó recursos generalizados de resistencia.

Quizá el motivo de este sesgo hacia la psicopatología que orientaron las investigaciones en las ciencias de la salud y que ha llevado a negar las características positivas del ser humano haya sido la necesidad de resolver numerosas problemáticas de salud mental (Seligman \& Csiksentmihalyi, 2000; Contini, 2001). Así también, este enfoque psicopatológico ha contribuido a asumir una posición pesimista de la condición humana. De este modo, características tales como el optimismo, la creatividad, la sabiduría o el sentido del humor no han sido objeto de estudio (Vera Poseck, 2006). Se agregará también la capacidad para vincularse satisfactoriamente con otros, esto es las HHSS que constituyen fuente de disfrute. Éstas tampoco han sido indagadas a fondo, en particular en la infancia y adolescencia, etapas cruciales en la adquisición de las mismas. Afortunadamente, se asiste en los últimos años a un interés creciente por investigar las HHSS. Tal cambio de posición, ocurrió al comprobarse que resulta más eficaz encarar programas de intervención para el desarrollo y fortalecimiento de las HHSS - en especial en niños y adolescentes - que trabajar solo sobre las disfunciones de las mismas. Sería inadecuado desde el punto de vista de la prevención primaria intervenir solo cuando la sintomatología ya está instalada, vale decir, cuando se hacen presentes conductas de agresividad y violencia, o su polo opuesto la inhibición y el aislamiento.

En esta línea, gran parte de las investigaciones buscan la manera de prevenir el desarrollo de trastornos en sujetos potencialmente vulnerables (Vera Poseck, 2006), lo que aún no se ha podido lograr totalmente. Seligman y Csikszentmihalyi (2000) afirman que de hecho, los mayores progresos en prevención se han logrado en la construcción de competencias. Para el caso que nos ocupa, se trataría del logro de competencias sociales. Lo que se ha podido demostrar, es que el sujeto tiene fortalezas que actúan como amortiguadores contra los trastornos mentales. También se podría decir que operan como factores protectores. En la literatura se citan algunas fortalezas tales como el optimismo, la esperanza, la perseverancia, la 
sabiduría, el liderazgo o el sentido del humor (Casullo, 2007; Seligman, 2003; Vera Poseck, 2003) y las habilidades de interacción social pueden ser consideradas en este mismo sentido.

Se puede afirmar entonces que la llamada Psicología Positiva retoma las ideas de la psicología humanista acerca de la necesidad del estudio de la 'parte positiva' de la existencia humana y "ha aportado un sólido soporte empírico y científico a esta parte descuidada de la psicología” (Vásquez, 2006, p.4). Por otra parte, tiene como objetivo mejorar la calidad de vida y prevenir la aparición de trastornos mentales (Vera Poseck, 2006; Casullo, 2007).

En síntesis, La Psicología Positiva propone tomar en cuenta el capital psíquico con el que cuenta cada sujeto. En relación al tema que nos ocupa, se dirá que las HHSS constituyen parte de ese capital. Como señala con acierto Casullo (2007), sin desconocer que la psicología debe atender las diversas problemáticas psicopatológicas, debe preocuparse por tener una mirada integradora que procure el desarrollo de un proyecto saludable, tenga en cuenta las fortalezas del sujeto y no solo el malestar, propio del antiguo modelo médico.

\section{Por qué estudiar las HHSS en la adolescencia temprana}

El paso de la niñez a la adolescencia es una de las transiciones evolutivas más trascendentales del ciclo vital. Los cambios físicos y psíquicos implican una modificación del rol del adolescente, en cómo se ve a sí mismo, cómo percibe al mundo y cómo es visto por los otros (Martínez Guzmán, 2007).

En esta transición, la preadolescencia o adolescencia inicial (Griffa \& Moreno, 2005) constituye un momento crucial al iniciarse la reestructuración del esquema corporal y la reorganización de la personalidad. A estos cambios, se suman los escolares, ya que también, es el periodo en el cual el adolescente debe hacer un pasaje de la escuela primaria a la escuela de nivel medio.

Si bien las teorías más recientes afirman que adolescencia no es sinónimo de psicopatología (Ávila Espada, Jiménez Gómez y González Martínez 1996; Casullo y Castro Solano, 2000; Casullo y Fernández Liporace, 2001; Casullo et al. 2002; Contini, Figueroa, Cohen Imach y Coronel, 2003; Contini, 2006), no se puede negar que tanto la complejidad de las sociedades postindustriales actuales, como la pobreza, generan presiones, que provocan estrés en el adolescente (Lucio, Barcelata, Durán \& Villafranca, 2004). En particular, el contexto social y económico de Latinoamérica caracterizado por la inequidad y las desventajas de toda índole para los más pobres, hacen que este pasaje pueda tornarse problemático. La precariedad social y familiar de estos adolescentes hace imprescindible el diagnóstico de los recursos cognitivos y afectivos con los que cuentan, para delinear así programas de intervención que promuevan estrategias y habilidades protectoras para su desarrollo. Se está haciendo referencia a las HHSS necesarias para el aprendizaje escolar, la eficacia en las relaciones interpersonales y la adaptación activa a situaciones sociales. 


\section{Síntesis}

Se ha tomado como objeto de estudio a las habilidades sociales, con particular referencia a su valor en la adolescencia temprana. Numerosas investigaciones permitieron confirmar que las habilidades cognitivas evaluadas por medio de pruebas de CI no hacen posible establecer pronósticos certeros sobre el éxito en la vida cotidiana; por ello, en las últimas décadas han tomado relevancia las investigaciones sobre las HHSS.

Se deslindaron los conceptos de inteligencia social, habilidades sociales, competencia social, asertividad y comportamiento adaptativo. Se caracterizaron las HHSS, y se describieron estilos de habilidades sociales identificando los denominados asertivo, no asertivo y agresivo. Igualmente, se hizo referencia a teorías explicativas de las mismas. Se dejaron planteados interrogantes sobre la posible interacción entre HHSS, personalidad y ajuste psicosocial, tema que merece un estudio en mayor profundidad por sus implicancias en el campo de la clínica psicológica. En tal sentido, el modelado temprano de HHSS positivas, asertivas, en la relación asimétrica madrehijo, y posteriormente entre pares, parece tener incidencia notable en la configuración de una personalidad saludable, por lo que el abordaje de las mismas desde la temprana infancia sería una clave en la atención primaria de la salud.

Igualmente, se enfocó el estudio de las HHSS desde el marco de la Psicología Positiva. Ésta plantea la necesidad de descentrar el interés en la psicopatología y valorar las fortalezas y el capital psíquico del sujeto. En tal sentido, al analizar las funciones que cumplen las HHSS se señaló que permiten al niño /adolescente el conocimiento de sí mismo y de los demás; el desarrollo de conductas de reciprocidad, de colaboración, el intercambio en el control de la relación; el desarrollo de estrategias sociales de negociación y de acuerdos; el autocontrol de la propia conducta en función del feed-back que recibe de los otros. Las HHSS también cumplen la función de apoyo emocional, de ser una fuente de disfrute, hacen posible el aprendizaje del rol sexual y el aprendizaje de valores. De este análisis, se puede concluir que las relaciones interpersonales y, en particular con los pares, es decididamente importante en el proceso de desarrollo y operarían como un factor protector para el logro de la salud en la adolescencia.

Se puso especial énfasis en el abordaje de las HHSS en la preadolescencia o adolescencia temprana por cuanto el tránsito a la adultez es una de los más trascendentes del ciclo vital, tanto para los que han nacido en sociedades postindustriales, como para los que viven bajo condiciones de pobreza. Ambos contextos generan estrés al adolescente.

Tales HHSS son esenciales no sólo por su dimensión relacional, sino por su influencia en otras áreas de la vida del adolescente. Está comprobado que aquellos niños y/o adolescentes que manifiestan dificultades para relacionarse o ser aceptados por sus compañeros de aula, tienden a presentar problemas a largo plazo vinculados con la deserción escolar, los comportamientos violentos y las perturbaciones psicopatológicas en la vida adulta (Ison, 1997; Arias Martínez \& Fuertes Zurita, 1999; Michelson, Sugai, Wood \& Kazdin, 1987; Monjas Casares \& González Moreno, 1998). 
Por lo tanto, es muy importante para la psicología poder identificar las disfunciones en las HHSS vinculadas a los comportamientos violentos, o a los comportamientos de aislamiento. Estos últimos, se presentan asociados con la depresión y a veces con el suicidio, de cuya creciente emergencia da cuenta la consulta tanto de la familia, como de instituciones escolares y de los mismos Mass media. Desde todos estos ámbitos, se necesita tener un mayor y fundado conocimiento de este aspecto de la vida psíquica del adolescente.

El diagnóstico oportuno de los recursos cognitivos y afectivos con los que cuenta el adolescente - o bien sus disfunciones - permitirá delinear programas de intervención que promuevan habilidades protectoras para su desarrollo. Las HHSS, al interior del paradigma de la Psicología Positiva, operan como un factor protector y, por ende constituyen un recurso salugénico en la adolescencia temprana.

\section{Bibliografía}

Antonovsky, A. (1979). Health, stress and coping: new perspectives on mental and Physical Well-Being. San Francisco: Jossey-Bass.

Antonovsky, A. (1988). Unraveling the mystery of health. How people manage stress and stay well. San Francisco: Jossey-Bass Publishers.

Arias Martínez, B. \& Fuertes Zurita, J. (1999). Competencia social y solución de problemas sociales en niños de educación infantil: un estudio observacional. Mente y Conducta en situación educativa. Revista electrónica del Departamento de Psicología Universidad de Valladolid, 1 (1), 1-40.

Argyle, M. (1983). The psychology of interpersonal behavior, $4^{\circ}$ edition, Harmondsworth. Penguin Books.

Ávila-Espada, A., Jiménez-Gómez, F. \& González Martínez, M. (1996). Aproximación psicométrica a los patrones de personalidad y estilos de afrontamiento del estrés en la adolescencia: perspectivas conceptuales y técnicas de evaluación en Casullo comp., Evaluación psicológica en el campo de la salud. Barcelona: Paidós-Ibérica.

Ayala Velásquez, H., Pedroza Cabrera, F., Morales Chainé, S., Chaparro Caso-López, A. \& Barragán Torres, N. (2002). Factores de riesgo, factores protectores y generalización del comportamiento agresivo en una muestra de niños en edad escolar. Salud Mental, 25 (3), 27-40.

Ballester, R. \& Gil Llario, M. (2002). Habilidades sociales. Madrid: Síntesis.

Bandura, A. \& Walters, R (1978). Aprendizaje social y desarrollo de la personalidad. Madrid: Alianza.

Blanco, A. (1982). Evaluación de las habilidades sociales. En R. Fernández Ballesteros \& Carrobles, J. (Eds.). Evaluación conductual. Madrid: Pirámide. 
Brailo, S., Seguel, X. \& Montenegro, H. (1987). Prevalencia de trastornos psíquicos en la población escolar de Santiago de Chile. Acta Psiquiátrica y Psicológica de América Latina, 33, 316-325.

Caballo, V. (1987). Teoría, evaluación y entrenamiento de las habilidades sociales. Valencia: Promolibro.

Caballo, V. (1991). El entrenamiento en habilidades sociales. En V. Caballo (comp). Manual de técnicas de terapia y modificación de conducta. Madrid: Silgo XXI.

Caballo, V. (1993). Relaciones entre diversas medidas conductuales y de autoinforme de las habilidades sociales. Psicología Conductual, 1, 73-99.

Caballo, V. (2000). Manual de evaluación y entrenamiento de habilidades sociales. Madrid: Siglo XXI.

Cantor, N. \& Kihlstrom, J. (1987). Personality and social intelligence. Englewood Cliffs, NJ: Prentice-Hall.

Casullo, M. M. \& Castro Solano, A. (2000). Evaluación del Bienestar Psicológico en estudiantes adolescentes argentinos. Revista de Psicología. Lima: Pontificia Universidad Católica del Perú. XVIII, (2), 35-68.

Casullo, M. M. \& Fernández Liporace, M. (2001). Estrategias de afrontamiento en estudiantes adolescentes. Revista del Instituto de Investigaciones. Buenos Aires, Facultad de Psicología, 6 (1), 25-49.

Casullo, M. M. Brenlla, M, Castro Solano, A., Cruz, S., González, R., Maganto, C. Martín, M, Martínez, P, Montoya, I \& Morote, R. (2002). Evaluación del bienestar psicológico en Iberoamérica. Bs. As. : Paidós.

Casullo, M.M. (comp.) (2007). Prácticas en Psicología Positiva. Buenos Aires: Lugar.

Cervone, N., Luzzi, A., Slapak, S. \& Samaniego, V. (2000). Estudio descriptivo de una población infantil derivada para su asistencia clínica en un centro comunitario. VII Anuario de Investigaciones, 203-222.

Connor, J., Dann, L. \& Twentyman, C. (1982). A self-report measure of assertiveness in young adolescents. Journal of Clinical Psychology, 38, 101-

Contini, N. (2001). Hacia un cambio de paradigma. De la psicopatología al Bienestar Psicológico. Acta Psiquiátrica y Psicológica de América Latina. 47(4): 306-315.

Contini, N. Figueroa, I., Cohen Imach, S. \& Coronel, P. (2003). Bienestar Psicológico y Estrategias de Afrontamiento en adolescentes escolarizados de Tucumán (13 y 14 años). Revista del Instituto de Investigaciones. Buenos Aires: Facultad de Psicología, UBA, 8, (2), 61-78.

Contini, N. (comp.) (2006). Pensar la adolescencia hoy. De la psicopatología al bienestar psicológico. Bs. As. : Paidós. 
Da Dalt de Mangione, E. \& Difabio de Anglat, H. (2002). Asertividad. Su relación con los estilos deductivos familiares. Interdisciplinaria: Buenos Aires: CIIPME, 19, 2, 119-140.

Dollard, J. \& Miller, N. (1977). Personalidad y Psicoterapia. Bilbao. DDB.

Fernández Ballesteros, R. (1994). Evaluación conductual hoy. Un enfoque para el cambio en psicología clínica y de la salud. Madrid: Pirámide.

Ford, M. E. \& Maher, M. A. (1998). Self-awareness and social intelligence. In M. D. Ferrari \& R. Sternberg (Eds), Self-awareness: Its nature and development). New York: Gilford Press, 191-218.

Gil, F. \& León Rubio, J (1998). Habilidades Sociales. Teoría, investigación e intervención. Madrid: Síntesis.

Gilbert, D. \& Connoly, J. (1995). Personalidad, habilidades sociales y psicopatología: un enfoque diferencial. Barcelona: Omega.

Goleman, D. (2000). La inteligencia emocional. Bs. As. : Vergara. 25a. edición

Gardner, H. (1993).Seven creators of the modern era. In J. Brockman (Ed), Creativity. New York: Simon \& Schuster, 28-47.

Gardner, H. (1994). Estructuras de la mente. La teoría de las inteligencias múltiples. México: Fondo de Cultura Económica.

Godoy, A., Gavino, A., Blanco, J., Martorell, M., Navarro, A. \& Silva, F. (1993). Escalas de Lugar de Control en Situaciones Académicas (ELC-A). En F. Silva \& C. Martorell (Dirs.), EPIJ: Evaluación de la personalidad infantil y juvenil. Madrid: MEPSA, 3779.

Griffa, M. \& Moreno, J. (2005). Claves para una Psicología del Desarrollo. Adolescencia. Adultez. Vejez. Buenos Aires: Lugar Editorial. Vol. 2.

Hops, H. \& Greenwood, C. R. (1988). Social skills deficits. En E. J. Mash y L. G. Terdal (Eds.) Behavioural assessment of childhood disorders. New York: Guildford Press, 2nd ed., 263-314.

Ison, M. \& Soria, E. (1997). Baremo de la Guía de Observación Comportamental para niños. Interdisciplinaria, 14 (1-2), 25-46.

Ison, M. S. (2004). Características familiares y habilidades socio-cognitivas en niños con conductas disruptivas. Revista Latinoamericana de Psicología, 36 (2), 257-268.

Kelly, J. (1987). Entrenamiento de las habilidades Sociales. Bilbao: Desclée de Brouwer.

Ladd, G. \& Asher, S. (1985). Social skill training and children's peer relations. In L. L'Abate \& M. A. Milan (Eds.), Handbook of Social Skills Training and Research. New York: Wiley, 219-244. 
León Rubio, J. \& Medina Anzano, S. (1998). Aproximación conceptual a las habilidades sociales en Habilidades Sociales. Teoría, investigación e intervención. Madrid: Síntesis. Lucio G.M., E., Barcelata, B., Durán, C. \& Villafranca, L. (2004). Sucesos de vida estresantes: un estudio transcultural de adolescentes venezolanos y mexicanos. Revista Iberoamericana de Diagnóstico y Evaluación Psicológica, 17, (1), 123-138.

Martinez Guzmán, M. (2007). Mirando al Futuro: Desafíos y Oportunidades Para el Desarrollo de los Adolescentes en Chile. Psykhe, 16 (1), 3-14.

Matson, J., Rotatori, A., \& Helsel, W. (1983). Development of a rating scale to measure social skills in children: the Matson evaluation of social skills with youngsters (MESSY). Behavior Research Therapy, 21 (49), 335-340.

McFall, R. (1982) A review and reformulation of the concept of social skills. Behavioural assessment, $N^{\circ} 4,1-33$.

Michelson, L., Sugai, D., Wood, R. \& Kazdin, A. (1987). Las habilidades sociales en la infancia: Evaluación y tratamiento. Barcelona: Martinez Roca.

Millon, T. (1969). Modern Psychopathology: A biosocial approach to maladaptive learning and functioning. Philadelphia: Saunders.

Millon, T. (1977). Millon Adolescent Personality Inventory (MAPI). Minneapolis: National Computer Systems.

Millon, T. (1990). Toward a new personology. An evolutionary model. New York: Wiley.

Millon, T. (1994). Millon Adolescent Clinical Inventory (MACI). Minneapolis: National Computer Systems.

Millon, T. (1998). Los trastornos de la personalidad más allá del DSM IV. Barcelona: Masson.

Monjas Casares, M. \& González Moreno, B. (1998). Las habilidades sociales en el currículo (Serie Colección N 146). España: Centro de Investigación y Documentación Educativa- CIDE. España, No 146.

Monjas Casares, M. (2000). Programa de enseñanza de habilidades de interacción social (PEHIS) para niños y niñas en edad escolar. Madrid: CEPE.

Morales, J. (1981). La conducta social como intercambio. Bilbao: DDB.

Neisser, U. (1979). The concept of intelligence, Intelligence, 3: 217-227.

Ortega, R. \& Monks, C. (2005). Agresividad injustificada entre preescolares. Psicothema, 17 (3), 453-458.

Paula Pérez, I. (2000). Habilidades sociales: educar hacia la autorregulación. Conceptualización, evaluación e intervención. Barcelona: ICE-Horsori. 
Pithod, A. (1993). Adaptación y estandarización de test para uso en comportamiento organizacional. Mendoza, Argentina. Archivos Centro de Investigaciones Cuyo (CIC). Polanyi, M. (1966). The tacit dimensions. Garden City. NY: Doubleday.

Salovey, P. \& Mayer, J. (1990). Emotional Intelligence. Imagination, Cognition and Personality, 9:185-211.

Samaniego, C. (1998). El Child Behavior Checklist: su estandarización y aplicación en un estudio epidemiológico. Problemas comportamentales y sucesos de vida en niños de 6 a 11 años de edad. Informe final. Beca UBACYT.

Samaniego, C. (2004). Prevalencia de trastornos psíquicos en población escolar de 6 a 11 años de edad. Memorias de las XI Jornadas de Investigación Psicología, sociedad y cultura. Facultad de Psicología, Universidad de Buenos Aires, Tomo II, 226-228.

Schön, D. (1983). The reflective practitioner: How professional think in action. NY: Basic Books.

Seligman, M. \& Csikszentmihalyi, M. (2000) Positive Psychology: an Introduction. American Psychologist, 55 (1): 5-14.

Seligman, M. (2003). La auténtica felicidad. Barcelona: Javier Vergara Editor.

Sternberg, R. (1985). Beyond IQ: A triarchic theory of human intelligence: New York: Cambridge University Press.

Sternberg, R. (1997). Inteligencia exitosa. Barcelona: Paidós.

Sullivan, H. (1953). The interpersonal theory of psychiatry. Nueva York: Norton.

Trower, P. (1986). Social skills training and social anxiety. En C. Hollin y P.

Trower (Eds.), Handbook of social skills training. Oxford: Pergamon Press.

Vásquez, C. (2006). La Psicología Positiva en perspectiva. Madrid: Colegio Oficial de Psicólogos. Papeles del Psicólogo. Vol. 27(1): 1-2. Recuperado de http://www.cop.es/ papeles el 6 de agosto de 2007.

Vera Poseck, B. (2006). Psicología Positiva. Una nueva forma de entender la psicología. Madrid: Colegio Oficial de Psicólogos. Papeles del Psicólogo. Vol. 27(1), 3-8. Recuperado de http://www.cop.es/papeles el 6 de agosto de 2007.

Wagner, R. \& Sternberg, R. (1985). Practical intelligence in real-world pursuits: The role of tacit knowledge. Journal of Personality \& Social Psychology, 49, 436-458.

Wolpe, J. (1977). Práctica de la terapia de conducta. México: Trillas. 
Psicodebate 9. Psicología, Cultura y Sociedad 